\title{
Imaging Cellulose Nanocrystals
}

\author{
Iulia Sacui $^{1}$, Henryk Szmacinski ${ }^{2}$, Daniel L. Blair ${ }^{3}$, Jeffery S. Urbach ${ }^{3}$, Mauro Zammarano ${ }^{1}$ and Jeffery \\ W. Gilman ${ }^{1}$ \\ 1. NIST, Material Science and Engineering Division, Gaithersburg, MD, USA. \\ 2. University of Maryland School of Medicine, Department of Biochemistry and Molecular Biology, \\ Baltimore, MD, USA. \\ 3. Georgetown University, Department of Physics, Washington, DC, USA.
}

Our project focuses on the characterization of cellulose nanocrystals (CNC) and their use as nanoparticle reinforcing agents in sustainable composites. CNC are an abundant, sustainable natural polymer that are biodegradable and easy to functionalize. CNC offer a high degree of crystallinity, high modulus (138 $\mathrm{GPa}$ for tunicate), and low density $\left(1.5 \mathrm{~g} / \mathrm{cm}^{3}\right)$ [1]. The main focus of the project is to determine the inherent stability and state of dispersion of $\mathrm{CNC}$ in aqueous solutions and determine the effects of varying morphology, surface charge, and aspect ratio on the dispersion of CNC in aqueous solutions.

Initial cellulose sources (wood, bacteria, and tunicate) were hydrolyzed to give a variety of CNC morphologies and dispersion behaviors, which can be imaged using atomic force microscopy (AFM). Of the hydrolysis techniques used, acid hydrolysis produces rigid rod nanocrystals by cutting through the amorphous regions of the fibers perpendicular to their axis (Figure 1b), while enzyme hydrolysis breaks apart the fibers along their axis, leading to a network of nanofibrils (Figure 1a) [2]. In addition, sulfuric acid hydrolysis of cellulose not only breaks apart the larger fibers into $\mathrm{CNC}$, but also functionalizes the $\mathrm{CNC}$ with sulfate groups. These negatively charged sulfate groups facilitate the dispersion of CNC in aqueous solution and on the AFM substrate, allowing for ease in $\mathrm{CNC}$ length measurements and length separation.

After initial AFM imaging, the CNC were then functionalized with a fluorescent dye to allow for optical imaging of $\mathrm{CNC}$ in solution and in a composite. Fluorescent dye, 5-(4,6-dichlorotriazinyl) aminofluorescein (DTAF), was reacted with the primary hydroxyl group on the cellulose surface under basic conditions [3]. The reaction was then purified using a vigorous cross flow filtration method to remove any unreacted dye. The final purified DTAF-labeled fibers were examined using confocal fluorescence lifetime imaging microscopy (FLIM) to determine if unattached dye remained in the samples after purification [4]. For the wood CNC, the calculated lifetime image (Figure 2a) displayed uniform lifetime values with a narrow lifetime distribution as shown in Figure $2 \mathrm{~b}$. The observed lifetime results indicate that DTAF is in a homogeneous environment, as is expected for a clean (purified) sample. If the composite included unreacted DTAF, it would display a much broader or bimodal distribution indicating a heterogeneous dye environment. FLIM data is highly informative on the environment heterogeneity, thus on the chemical structure of the composite.

Once the purity of the fluorescently labeled CNC was determined, the CNC were then imaged in solution under shear, using a specially designed confocal-rheometer [5]. This instrument is a combination of an inverted confocal microscope and parallel plate rheometer, enabling imaging of samples in solution under shear. In this case, the confocal-rheometer was used to image the dispersive ability of the negatively charged sulfate $\mathrm{CNC}$ as compared to the neutrally charged CNC, which tended to aggregate under low shear. 
Future research in the area will focus on incorporating $\mathrm{CNC}$ in a polymer matrix, imaging the composite by confocal microscopy, and determining the effect of the differences in CNC properties on the mechanical properties of the composite.

\section{References:}

[1] Habibi, Y., et al Chemical Reviews 110 (2010), p. 3479-3500.

[2] Paakko, M., et al Biomacromolecules 8 (2007), p. 1934-1941.

[3] Stead, C. V., Dyes and Pigments 3 (1982), p. 161-171.

[4] Wallrabe, H., Periasamy, A., Current Opinion in Biotechnology 16 (2005), p. 19-27.

[5] Holland, C., et al Soft Matter 8 (2012), p. 2590-2594.

Disclosure: This work was carried out by the National Institute of Standards and Technology (NIST), an agency of the U.S. government, and by statute is not subject to copyright in the United States.

Acknowledgment: Financial support was provided by the National Institute of Standards and Technology and the Air Force office of Scientific Research under Award No. F1ATA00236G002.

1a)

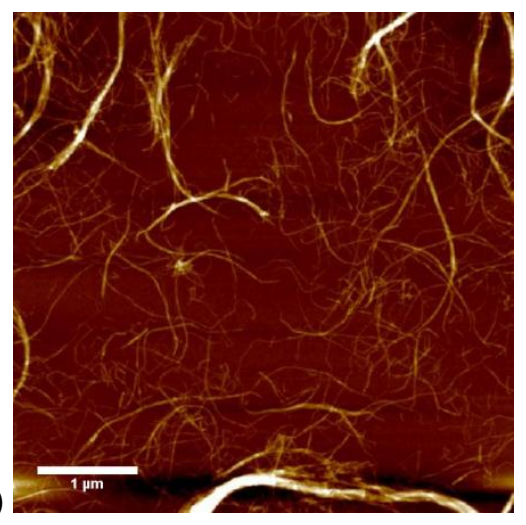

1b)

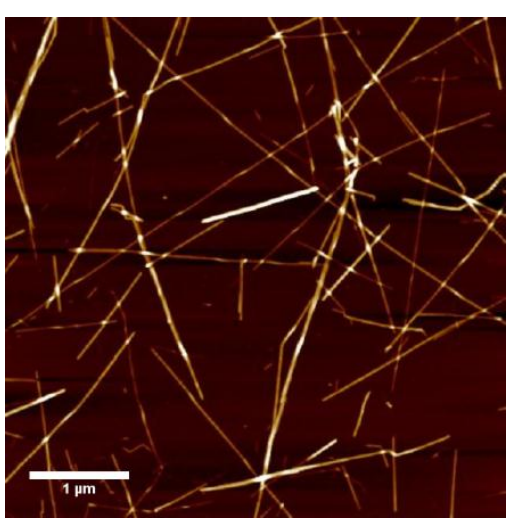

Figure 1. AFM image of (a) wood-enzyme hydrolysis CNCs and (b) tunicates-sulfuric acid hydrolysis CNCs on lysine coated mica substrate.

2a)

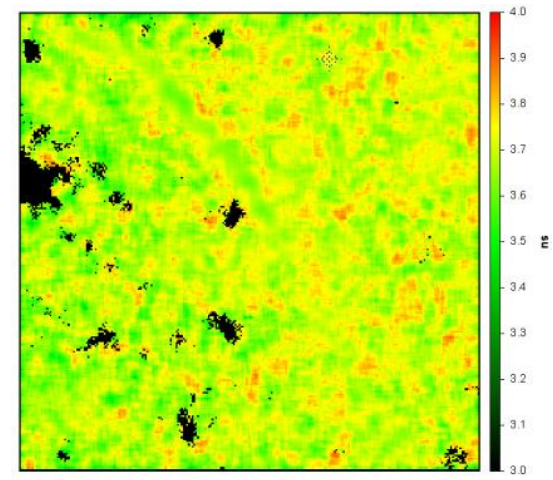

Figure 2. (a) Lifetime image of fluorescently labeled (DTAF) wood-enzyme hydrolysis CNCs (image size $300 \mu \mathrm{m} \times 300 \mu \mathrm{m})$ and (b) graph of lifetime distribution (3.70 $\mathrm{ns} \pm 0.06 \mathrm{~ns})$. 